\section{DNA profiling on trial}

SIR - D. Balding and P. Donnelly ${ }^{1}$ are correct in their point that the match probability arising from a DNA profile is not the same as the probability of innocence of the suspect - the probability of innocence depends upon the totality of the evidence. But their hypothetical calculation to illustrate the difference between the two probabilities is not helpful.

They consider a case in which an individual has a match with a scene-of-crime DNA, which can be calculated to have a probability of one in a million, but this individual has, on the basis of the evidence other than the DNA profile, approximately a one in a half million chance of being guilty. Balding and Donnelly conclude, correctly on the basis of their assumptions, that the suspect has, when the DNA evidence is included, about a one in three chance of being innocent. Their figure of half a million, however, is an entirely arbitrary choice, and is not relevant to real cases where DNA profiling has been used as evidence. In a typical case, a match arises because a suspect's DNA has been profiled and compared to that from a DNA sample from the scene of a crime. Forensic scientists have better things to do with their time than to screen systematically, with DNA profiling, individuals who have only a one in half a million chance of being guilty of the crime that is being investigated.

In the future it may be true that suspects emerge through the screening of databases containing very many individuals, whose profiles were not collected in connection with the crime being investigated. In these circumstances it may indeed be true that suspects have very low prior probabilities of guilt, and this should be incorporated in the standard way using Bayes' formula in the assessment of the final probability of guilt.

\section{John Brookfield}

Department of Genetics,

University of Nottingham,

Nottingham NG7 2UH, UK

SIR - The account by Balding and Donnelly ${ }^{t}$ of the use of DNA evidence in courts cannot be correctly accessed from the citations given by the authors. As of 18 March 1994, DNA evidence has been considered in 139 cases in US courts and admitted in 124 of them. Some of the remands or exclusions of the remaining 15 cases are also under re-examination. In no case was DNA the sole evidence implicating the suspect in the crime, unlike the situation depicted by Balding and Donnelly.

The authors' concern about 'false match' errors does not acknowledge that most forensic case analyses provide sufficient scope to check whether errors could have occurred because the band intensity, size and other attributes of autoradiographs are indicative enough of which sample is the evidentiary one and which bands come from a relatively fresh sample. Furthermore, opportunities for retesting the samples from suspect, victim and evidence materials have never been used to document 'false match' errors.

I do not suggest that forensic laboratories cannot and do not make mistakes, but reviews of databases generated by forensic laboratories suggest that a match beyond five loci is virtually improbable for any cosmopolitan population ${ }^{2}$, and even in a highly inbred population a multilocus DNA match between close relatives is an exception rather than the rule ${ }^{3}$. For example, in reviewing more than 60 DNA evidence cases, I found that the general forensic laboratories produce autoradiographs that meet current scientific standards. In no two cases are the profiles even close to each other, and in every case no individual was found in a large database (more than 10,000 individual records) with a matching profile. A simple probability calculation would show that this cannot result from DNA profiles with 'unsafe' small reported frequencies.

Rhetorical criticisms of DNA testing can only generate confusion among judges and jurors alike. Significant effects of population substructure or not, the rarity of any specific hypervariable multilocus DNA profile is a biological fact. Whether or not a relative of the suspect is the perpetrator can be examined with a simple test without statistical arguments such as discussed by Balding and Donnelly.

\section{Ranajit Chakraborty}

Centerfor Demographic

and Population Genetics,

University of Texas

Houston Health Science Center,

PO Box 20334, Houston, Texas 77225, USA

SIR - Balding and Donnelly' consider that courts have been misled about the strength of DNA evidence. But although they make the correct distinction between the probability that a particular individual has the DNA profile in question and the probability that he has this profile given that the defendant has it, they overstate the distinction. For a single locus with allelic frequencies of 0.1 (their figures), the expected frequency of any heterozygote is 0.02 while the conditional frequency in a population for which the measure

\footnotetext{
1. Balding, D. J.\& Donnelly, P. Nature 368, 285-286 (1994).

2. Risch. N J \& Devlin B Science 255,717-720 (1992)

3. Chakraborty. R. \& Jin. L. Hum. Biol. 65, 875-895 (1993).

4. Cockerham, C. C. Genetics 69, 235-246(1971).

5. Brookfield, J. Heredity 69, 91-100 (1992).

6. Morton, N. E. Proc. natn. Acad. Sci. U.S.A. 89, 2556-
} 2560 (1992). of population structure $F_{S T}=0.001$ is the very similar 0.0203 (ref. 4 ). The difference between their figures of $10^{-7}$ and "might be $10^{-5}$ or more" corresponds to an $F_{S T}$ of 0.05 or more. It is clear that 0.001 is a much more realistic value than 0.05 (refs $5,6)$.

\section{B.S. Weir}

Department of Statistics,

North Carolina State University,

Raleigh, North Carolina 27695-8203, USA

\section{Optimismabounds}

SIR - In commenting on Kay Davies's decision not to take up her post as director of the Medical Research Council (MRC)'s new Clinical Sciences Centre (CSC), you reported a number of comments casting doubt on the future of research in the new Hammersmith Hospitals Trust (Nature 369, 92; 1994).

Although we are disappointed at Davies's decision, we remain optimistic about the future of research in the new trust. In particular:

- The MRC remains fully committed to the CSC, a replacement for Kay Davies is now being sought and the new laboratory building opens in June.

- The Royal Postgraduate Medical School, which has attracted the highest ratings in recent reviews by the Higher Education Funding Council for England, the Department of Health and so on, remains at Hammersmith Hospital.

- The government's formula for funding the excess costs of postgraduate research is satisfactory.

- The new trust, one of the largest in the National Health Service, is the dominant service provider in west London, and has a powerful concentration of specialist services that will provide the patient flow required to sustain high quality research. The trust had adopted the academically led clinical directorate structure, which was one of the foundations of Hammersmith's success.

- The combination of Charing Cross with Hammersmith has brought additional strength in several important areas, notably neurosciences, cancer and rheumatology.

For these reasons we do not share the pessimistic tone of your article. The new Hammersmith Trust, with its associated postgraduate and undergraduate medical schools and institutes, is already a powerful force in research, service and teaching. Now the uncertainty about the site is behind us, we can build for the future.

\section{Christopher Bland}

(Chairman, Hammersmith Hospitals Trust)

Colin Dollery

(Dean)

Royal Postgraduate Medical School, Hammersmith Hospital,

Du Cane Road, London W12 ONN, UK 